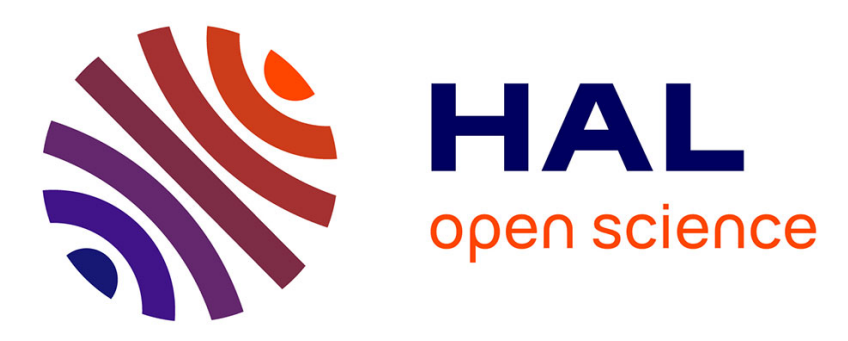

\title{
A solution of torsional problem by energy method in case of anisotropic cross-section
}

Pascal Swider, Jérôme Briot, Erik Estivalèzes

\section{To cite this version:}

Pascal Swider, Jérôme Briot, Erik Estivalèzes. A solution of torsional problem by energy method in case of anisotropic cross-section. Archive of Applied Mechanics, 2011, 81 (6), pp.801-808. 10.1007/s00419-010-0450-7 . hal-01588079

\section{HAL Id: hal-01588079 \\ https://hal.science/hal-01588079}

Submitted on 15 Sep 2017

HAL is a multi-disciplinary open access archive for the deposit and dissemination of scientific research documents, whether they are published or not. The documents may come from teaching and research institutions in France or abroad, or from public or private research centers.
L'archive ouverte pluridisciplinaire HAL, est destinée au dépôt et à la diffusion de documents scientifiques de niveau recherche, publiés ou non, émanant des établissements d'enseignement et de recherche français ou étrangers, des laboratoires publics ou privés. 


\section{Open Archive TOULOUSE Archive Ouverte (OATAO)}

OATAO is an open access repository that collects the work of Toulouse researchers and makes it freely available over the web where possible.

This is an author-deposited version published in : http://oatao.univ-toulouse.fr/ Eprints ID : 18175

To link to this article : DOI:10.1007/s00419-010-0450-7

URL : $\underline{\text { http://dx.doi.org/10.1007/s00419-010-0450-7 }}$

To cite this version : Swider, Pascal and Briot, Jérôme and Estivalèzes, Erik $A$ solution of torsional problem by energy method in case of anisotropic cross-section. (2011) Archive of Applied Mechanics, vol. 81 ( ${ }^{\circ}$ 6). pp. 801-808. ISSN 0939-1533

Any correspondence concerning this service should be sent to the repository administrator: staff-oatao@,listes-diff.inp-toulouse.fr 
Pascal Swider · Jérôme Briot • Erik Estivalèzes

\title{
A solution of torsional problem by energy method in case of anisotropic cross-section
}

\begin{abstract}
We proposed an original method to investigate the problem of torsion of anisotropic cross-section. We implemented an energy method to calculate the stress function represented by infinite series of trigonometric functions adapted to rectangular cross-section. After validation, we implemented a parametric sensitivity study to investigate the influence of the cross-section aspect ratio and the anisotropy level on the stress function, the strain energy density and the torsion stiffness. The process showed a fast convergence with a very good accuracy. The model showed a potential interest for the experimental identification of anisotropic material properties.
\end{abstract}

Keywords Torsion $\cdot$ Stress function $\cdot$ Energy method $\cdot$ Anisotropic material properties

\section{Introduction}

The solution of torsion problems of beams has been investigated for many years using theoretical, analytical and numerical approaches. Exact and approximate solutions have been provided for isotropic cross-sections of simple shape $[14,17]$ but for complex geometries, numerical approaches such as relaxation methods $[1,13,15]$ or finite element methods $[5,9,18]$ have been applied.

In case of anisotropic constitutive materials, analytical solutions for isotropic multiphasic cross-sections of simple shape have been proposed by $[7,10]$. Monophasic cross-sections with slightly varying constitutive properties also called functionally graded materials were more recently investigated $[2,6,12]$. Solutions of the torsion problem in case of association of anisotropic material properties with complex cross-sections were found by implementing full numerical solutions such as relaxation methods [3] and finite element methods $[4,11,16]$.

We propose in the present paper an energy method to study the torsion problem of anisotropic crosssections. The solution was searched in the form of infinite series of trigonometric functions for the representation of stress function. The method was applied to a rectangular cross-section involving anisotropic shear modules. The analytical solution allowed implementing a parametric sensitivity study to investigate the influence of cross-section aspect ratio and anisotropy level on stress function, strain energy density and torsion stiffness. Among several aspects approached in the discussion, we distinguished a potential interest of the method for the experimental identification of anisotropic material properties.

P. Swid · E. Estivalèzes

IMFT UMR CNRS 5502, University of Toulouse, CHU Purpan, Amphithéâtre Laporte, Place Dr Baylac,

31056 Toulouse Cedex, France

J. Briot

Biomechanics Laboratory, Purpan University Hospital, Toulouse, France 


\section{Theory and method}

\subsection{Energy method}

We considered the anisotropic cross-section $s$ presented in Fig. 1a with the shear field $\left(\tau_{x y}, \tau_{x z}\right)$. Equilibrium equations (1a) and (1b) were satisfied since the shear field was independent of $x$. Equation (1c) allowed deriving shear from the stress function (or Prandtl function) $\phi(y, z)$, which verified Eq. (2) on $s$ and was nil on the boundary $\partial s$.

$$
\begin{array}{llll}
\frac{\partial \tau_{x y}}{\partial x}=0 & \text { (a) } \quad \frac{\partial \tau_{x z}}{\partial x}=0 & \text { (b) } \quad \frac{\partial \tau_{x y}}{\partial y}+\frac{\partial \tau_{x z}}{\partial z}=0 \quad \text { (c) } \\
\tau_{x y}=\frac{\partial \phi}{\partial z .} & \text { and } \quad \tau_{x z}=-\frac{\partial \phi}{\partial y} \text { with } \phi(y, z)=0 \text { along } \partial s
\end{array}
$$

By introducing the strain field $\left(\varepsilon_{x y}, \varepsilon_{x z}\right)$ and the shear modules $\left(\mu_{x y}, \mu_{x z}\right)$, the strain energy per unit of length was expressed by Eq. (3). The torsion torque $T$ was given by Eq. (4) after integration by parts on $s$ with $\phi(y, z)=0$ at the boundary.

$$
\begin{aligned}
U & =\frac{1}{2} \int_{s}\left(\tau_{x y} \varepsilon_{x y}+\tau_{x z} \varepsilon_{x z}\right) \mathrm{d} y \mathrm{~d} z=\frac{1}{2} \int_{s}\left(\frac{\tau_{x y}^{2}}{\mu_{x y}}+\frac{\tau_{x z}{ }^{2}}{\mu_{x z}}\right) \mathrm{d} y \mathrm{~d} z \\
& =\frac{1}{2} \int_{S}\left[\frac{1}{\mu_{x y}}\left(\frac{\partial \phi}{\partial z}\right)^{2}+\frac{1}{\mu_{x z}}\left(\frac{\partial \phi}{\partial y}\right)^{2} \mathrm{~d} y \mathrm{~d} z\right. \\
T & =\int_{s}\left(-\tau_{x y} \times z+\tau_{x z} \times y\right) \mathrm{d} y \mathrm{~d} z=2 \int_{s} \phi(y, z) \mathrm{d} y \mathrm{~d} z
\end{aligned}
$$

The total potential energy $V$ was by definition the algebraic sum of potential strain energy $U$ and the work $W$ of punctual, surface and volume loads [17]. The principle of virtual work stated that if the total potential energy was stationary; then, the first-order variation of this energy was zero for any virtual displacement. The symbol $\delta$ was used to emphasize the virtual character of the instantaneous variations, and virtual displacement was only a name for arbitrary infinitesimal multipliers, which obeyed the rules of differential calculus [8].

The principle of virtual work was applied giving to the stress function $\phi$ the virtual variation $\delta \phi$, vanishing at the boundary. At equilibrium, the virtual variation of the total potential energy was zero as expressed by Eq. (5).

$$
\delta V=\delta U-\delta W=0
$$

Equations (3) and (4) were used to derive the virtual variation of strain energy $\delta U$ and the virtual work of torque $\delta W$, the torsion angle per unit length being $\theta$. The variational symbol $\delta$ was before the integrals since given loads and actual stresses were held unchanged. Finally, the virtual variation $\delta V$ was expressed by Eq. (6).

$$
\left.\delta V=\delta \int_{s} \frac{1}{2} \frac{1}{\mu_{x y}}\left(\frac{\partial \phi}{\partial z}\right)+\frac{1}{\mu_{x z}}\left(\frac{\partial \phi}{\partial y}\right)^{2}\right]-2 \theta \phi \mathrm{d} y \mathrm{~d} z=0
$$

Equation (7) was verified whatever the virtual variation $\delta \phi$ of stress function, so the true expression of $\phi(y, z)$ was that which made zero the variation of the total potential energy $V$. When we searched an approximate solution, the problem of variational calculus was transformed into the search of a minimum of the energy function $V$. The stress function was taken in the form of an infinite series $\phi(y, z)=\sum_{i} a_{i} \phi_{i}$ with a basis of functions $\phi_{i}$ verifying the boundary condition $\phi_{i}=0$ on $\partial s$. The search of minimum conditions of energy function $V$ consisted in calculating the coefficients $a_{i}$, which were the solutions of $\partial V / \partial a_{i}=0$. 


\subsection{Application}

The method was applied to a rectangular cross-section with $y= \pm a$ and $z= \pm b$ as shown in Fig. 1b. The material properties were anisotropic $\left(\mu_{x y} \neq \mu_{x z}\right)$ and independent from $y$ and $z$. We used an infinite series of trigonometric functions to represent the stress function $\phi(y, z)$ as expressed by Eq. (7). This basis verified the boundary conditions in $\phi$ as far as conditions of symmetry, index $m$ and $n$ being odd.

$$
\phi(y, z)=\sum_{m} \sum_{n} a_{m n} \cos \left(\frac{m \pi y}{2 a}\right) \cos \left(\frac{n \pi z}{2 b}\right)
$$

The first derivatives of $\phi(y, z)$ in $y$ and $z$ were replaced into Eq. (6) and after integration on domain $s$, the minimum conditions $\partial V / \partial a_{m n}=0$ allowed coefficients $a_{m n}$ to be obtained as follows:

$$
a_{m n}=\frac{128 a^{2} b^{2} \mu_{x y} \mu_{x z}(-1)^{\left(\frac{m+n}{2}-1\right)} \theta}{\pi^{4} m n\left(m^{2} b^{2} \mu_{x y}+n^{2} a^{2} \mu_{x z}\right)}
$$

The stress field (9) was obtained by replacing the stress function (7) into Eq. (2) with $a_{m n}$ given by expression (8).

$$
\begin{aligned}
& \tau_{x y}(y, z)=-\sum_{m} \sum_{n} a_{m n} \frac{n \pi}{2 b} \cos \left(\frac{m \pi y}{2 a}\right) \sin \left(\frac{n \pi z}{2 b}\right) \\
& \tau_{x z}(y, z)=\sum_{m} \sum_{n} a_{m n} \frac{m \pi}{2 a} \sin \left(\frac{m \pi y}{2 a}\right) \cos \left(\frac{n \pi z}{2 b}\right)
\end{aligned}
$$

The distribution pattern of strain energy density $d U$ in the cross-section was obtained by replacing the stress field (9) into Eq. (3) before integration.

\subsection{Parametric sensitivity analysis}

The torsion stiffness $\mu J$, expressed by Eq. (10), was obtained from Eq. (4) after integration of stress function $\phi(y, z)$. We added two adimensioned coefficients $\alpha=\mu_{x y} / \mu_{x z}$ and $\beta=b / a$, to represent the anisotropy level and the geometric aspect ratio, respectively. We also expressed the linear dependence to $\mu_{x z}$ by introducing the function $f(\alpha, \beta, a, m, n)$ in Eq. (10).

$$
\mu J=\frac{T}{\theta}=2 \int_{s} \phi(y, z) \mathrm{d} y \mathrm{~d} z=\frac{4096 a^{4} \mu_{x z}}{\pi^{6}} \sum_{m} \sum_{n} \frac{\alpha \beta^{3}}{m^{2} n^{2}\left(\alpha \beta^{2} m^{2}+n^{2}\right)}=f(\alpha, \beta, a, m, n) \times \mu_{x z}
$$

The Taylor expansion at the first order was used to quantify the influence of $\alpha$ and $\beta$ on the relative variation $\Delta \mu J / \mu J$ of the torsion stiffness. As shown in Eq. (11), the variation was expressed as a function of perturbation $\delta_{\alpha}$ and $\delta_{\beta}$ associated with direct sensitivities $S_{\mu} J_{\alpha}, S_{\mu} J_{\beta}$, and crossed sensitivity $S_{\mu} J_{\alpha \beta}$. Expanded forms are detailed in the Appendix.

$$
\begin{aligned}
\Delta \mu J / \mu J & =S \mu J_{\alpha}+S \mu J_{\beta}+S \mu J_{\alpha \beta}+\mathrm{O}\left(\delta_{\alpha}^{2}, \delta_{\beta}^{2}\right) \\
\text { with } S \mu J_{\alpha} & =(1 / f) \frac{\partial f}{\partial \alpha} \delta_{\alpha} \quad S \mu J_{\beta}=(1 / f) \frac{\partial f}{\partial \beta} \delta_{\beta} \quad S \mu J_{\alpha \beta}=(1 / f) \frac{\partial^{2} f}{\partial \alpha \partial \beta} \delta_{\alpha} \delta_{\beta}
\end{aligned}
$$

By using the similar Taylor expansion, we calculated the relative variation of shear module $\mu_{x z}$ to $\alpha$ and $\beta$. We assumed that the torsion stiffness $\mu J$ was known and the sensitivities were established as follows:

$$
\begin{aligned}
S \mu_{x z \alpha} & =-S \mu J_{\alpha} \\
S \mu_{x z \beta} & =-S \mu J_{\beta} \\
S \mu_{x z \alpha \beta} & =-S \mu J_{\alpha \beta}+2 S \mu J_{\alpha} \times S \mu J_{\beta}
\end{aligned}
$$


Table 1 Torsion stiffness $\mu J\left(\mathrm{Nm}^{2}\right)$ from the calculation of stress function $\phi(y, z)$ using analytical methods ${ }^{1}$ [17], infinite series ${ }^{2}$ [10], finite difference scheme ${ }^{3}[3]$, finite element method ${ }^{4}[11]$ and proposed method ${ }^{5}$

\begin{tabular}{|c|c|c|c|c|c|c|c|}
\hline & \multirow[t]{2}{*}{ (1) } & \multirow[t]{2}{*}{ (2) } & \multirow[t]{2}{*}{ (3) } & \multirow[t]{2}{*}{ (4) } & \multicolumn{3}{|l|}{$(5)$} \\
\hline & & & & & $m, n=1$ & $m, n=1,3$ & $m, n=1,15$ \\
\hline$\mu J$ & 0.1406 & 0.1407 & 0.1388 & 0.1406 & $0.1331(-5 \%)$ & $0.1392(-1 \%)$ & $0.1406(0 \%)$ \\
\hline
\end{tabular}

Results were obtained for an isotropic square cross-section $(\alpha=1, \beta=1)$ with shear modulus $\mu_{x y}=100 \mathrm{MPa}$ and side $a=5 \times 10^{-3} \mathrm{~m}$

\section{Results}

The initial step was to check the convergence process in $(m, n)$ of torsion stiffness $\mu J$ expressed by Eq. (11). As shown in Fig. 2, the convergence was dependant upon the anisotropy coefficient $\alpha$ and the aspect ratio $\beta$ but series converged very rapidly, and no significant differences appeared for $m, n \geq 15$. It was noted that good approximations were obtained with only one term $m, n=1$ since discrepancies were less than $9 \%$ for $\alpha$ and $\beta$ varying from 0.5 to 1.5 .

The accuracy of the method was then evaluated using reference solutions obtained for a square crosssection with isotropic material properties. Here again, the output measure was the torsion stiffness. Reference solutions were obtained from calculations of the stress function by analytical methods [14,17], infinite series [10], finite difference schemes [3] and a finite element method [11]. Results in Table 1 show that the proposed method provided accurate results. When we chose the analytical reference by [17], discrepancies of $-5,-1$ and $0 \%$ were obtained for $m, n$ terms, respectively, equal to 1,5 and 15 . This results was corroborated by the distribution pattern of the stress function $\phi(y, z)$ plotted in Fig. 3. It showed a good agreement with two numerical methods from the literature $[3,11]$.

Torsion stiffness and strain energy density were interdependent since both were relied to the stress field. We plotted in Fig. 4 the distribution pattern of the strain energy density for $\alpha$ and $\beta$ varying from 0.5 to 1.5. With a square isotropic cross-section $(\alpha=1, \beta=1)$, the diagonal was axis of symmetry. In case of anisotropic material, other plots showed that patterns were more irregular with an asymmetry influenced by the aspect ratio. This point was confirmed when the relative variation of the torsion stiffness was calculated using Eq. (12). Figure 5 shows that the sensitivity of $\mu J$ to $\alpha$ ranged from 0.3 to $0.9 \%$, whereas the sensitivity to $\beta$ ranged from 1.5 to $2.7 \%$. Their ratio ranged from $3.2(\alpha=0.5, \beta=0.5)$ to $5.9(\alpha=1.5, \beta=1.5)$, as shown in Fig. 5d.

We plotted the shear fields $\left(\tau_{x y}, \tau_{x z}\right)$ for an isotropic square cross-section (Fig. 6a: $\alpha=1, \beta=1$ ) and for a thin anisotropic cross-section with $\mu_{x y} \ll \mu_{x z}$ (Fig. 6b: $\alpha=0.5, \beta=0.5$ ). The first shear field (Fig. 6a) showed two similar patterns for $\tau_{x y}$ and $\tau_{x z}$ with progressive gradients. On the contrary, the second shear field (Fig. 6b) showed a progressive distribution of $\tau_{x y}$ while significant concentration gradients of $\tau_{x z}$ were located at the right end of the cross-section. This was due to the anisotropy level, which was favourable to $\mu_{x z}$. The effect on the strain energy density was attenuated because of the low aspect ratio $(\beta=0.5)$, as shown in Fig. 4d. It was observed that greater magnitudes of shear stress tended to migrate toward the upper end and vertical symmetry axis of the cross-section.

\section{Discussion and conclusion}

Our aim was to implement a phenomenological model to investigate torsion problem and complicated effects due to coupling between geometry and anisotropy. The semi-analytical resolution based upon an energy approach allowed this goal to be reached. We observed that the aspect ratio of the cross-section played a significant role on strain energy distribution and torsion behaviour. It proved to have greater influence than anisotropy, and this was particularly significant with thin sections.

In our developments, material properties were varying in direction but they were independent from the location. The method could be extended to location and orientation-varying materials such as composite or graded materials, assuming the governing laws of such variations were known. In that case, the integration of strain energy in Eq. (4) should be adapted. In case of a more complicated problem in terms of geometry, material distribution and material properties, this approach would find limitations thus complete numerical approach such as FE resolution would be required. 

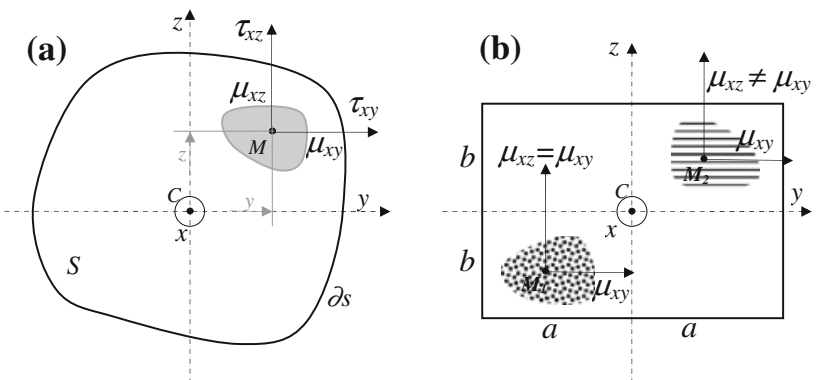

Fig. 1 Model of torsion problem. a The cross-section involved to shear modules $\mu_{x y}$ and $\mu_{x z}$ independent from point $M$ location; $x$ was the longitudinal axis of the beam. b Application to a prismatic cross-section with $y= \pm a$ and $z= \pm b$. $M_{1}$ represented a transversely isotropic material with isotropy plane in the cross-section $\left(\mu_{x y}=\mu_{x z}\right) ; M_{2}$ represented the same material with a $90^{\circ}$ rotation around axis $z\left(\mu_{x y} \neq \mu_{x z}\right)$

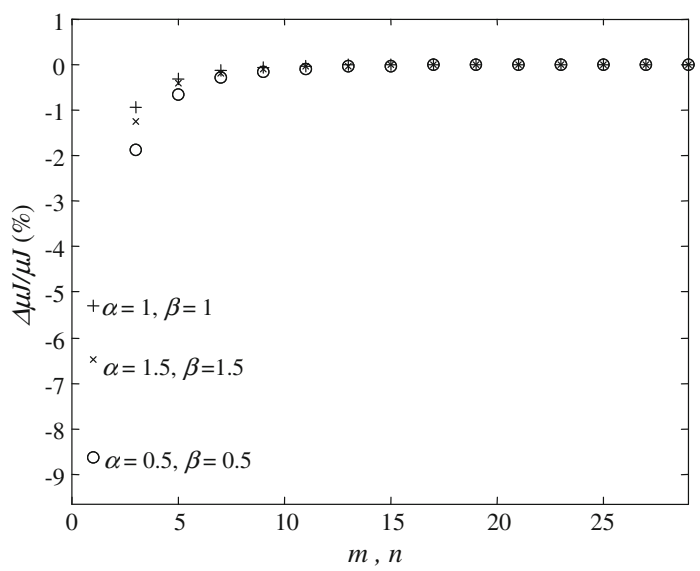

Fig. 2 Influence of index $m, n$ of infinite trigonometric series on the convergence process of torsion stiffness. The process was evaluated for a square isotropic cross-section $(+: \alpha=1, \beta=1)$, a thin anisotropic cross-section (o: $\alpha=0.5, \beta=0.5)$ and a thick anisotropic cross-section $(\times: \alpha=1.5, \beta=1.5)$. Shear modulus $\mu_{x y}=100 \mathrm{MPa}, a=5 \times 10^{-3} \mathrm{~m}$
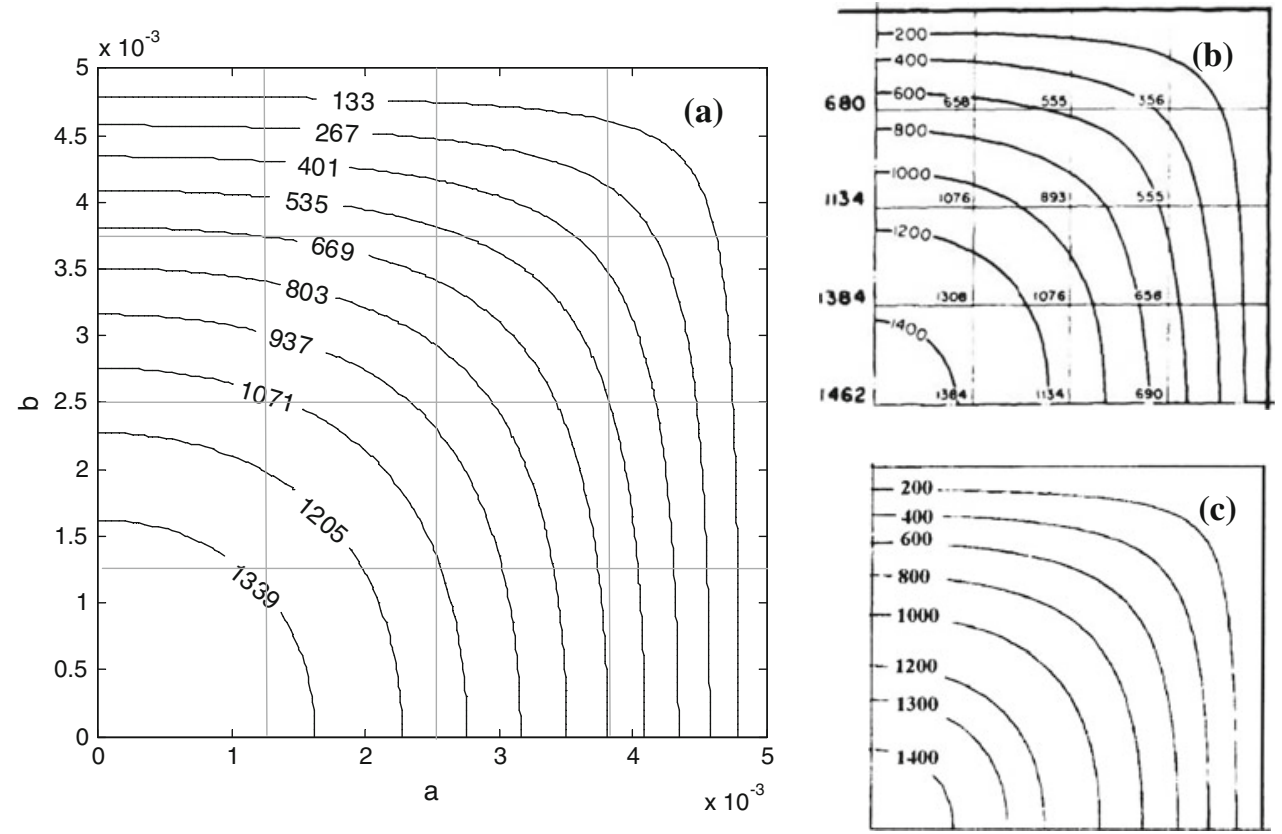

Fig. 3 Distribution patterns of stress function $\phi(y, z)(\mathrm{Pa} / \mathrm{m})$ calculated for a square isotropic cross-section $(\alpha=1, \beta=1)$. a Proposed method; b Ely [3]; c Pereira [11]. Shear modulus $\mu_{x y}=100 \mathrm{MPa}$, side $a=5 \times 10^{-3} \mathrm{~m}$ 
(b)

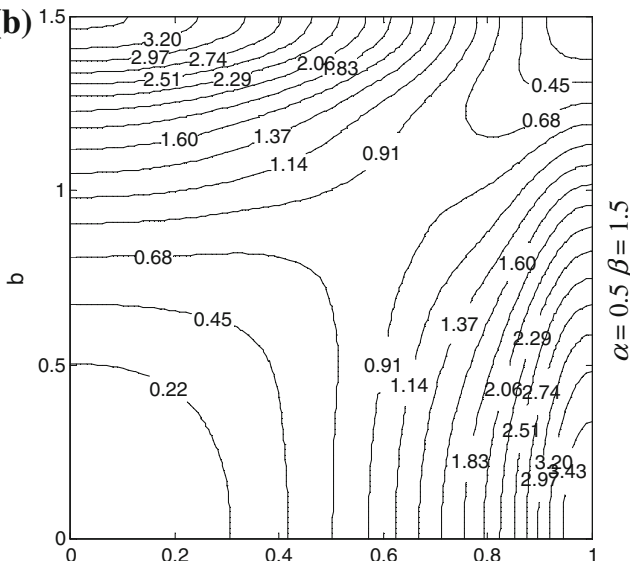

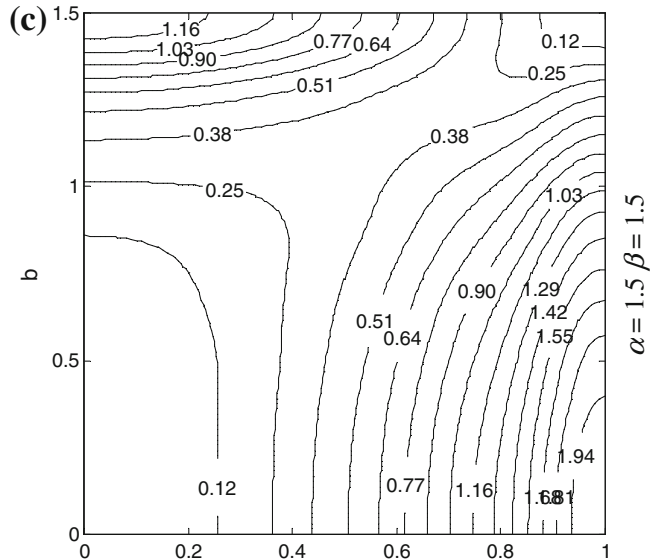
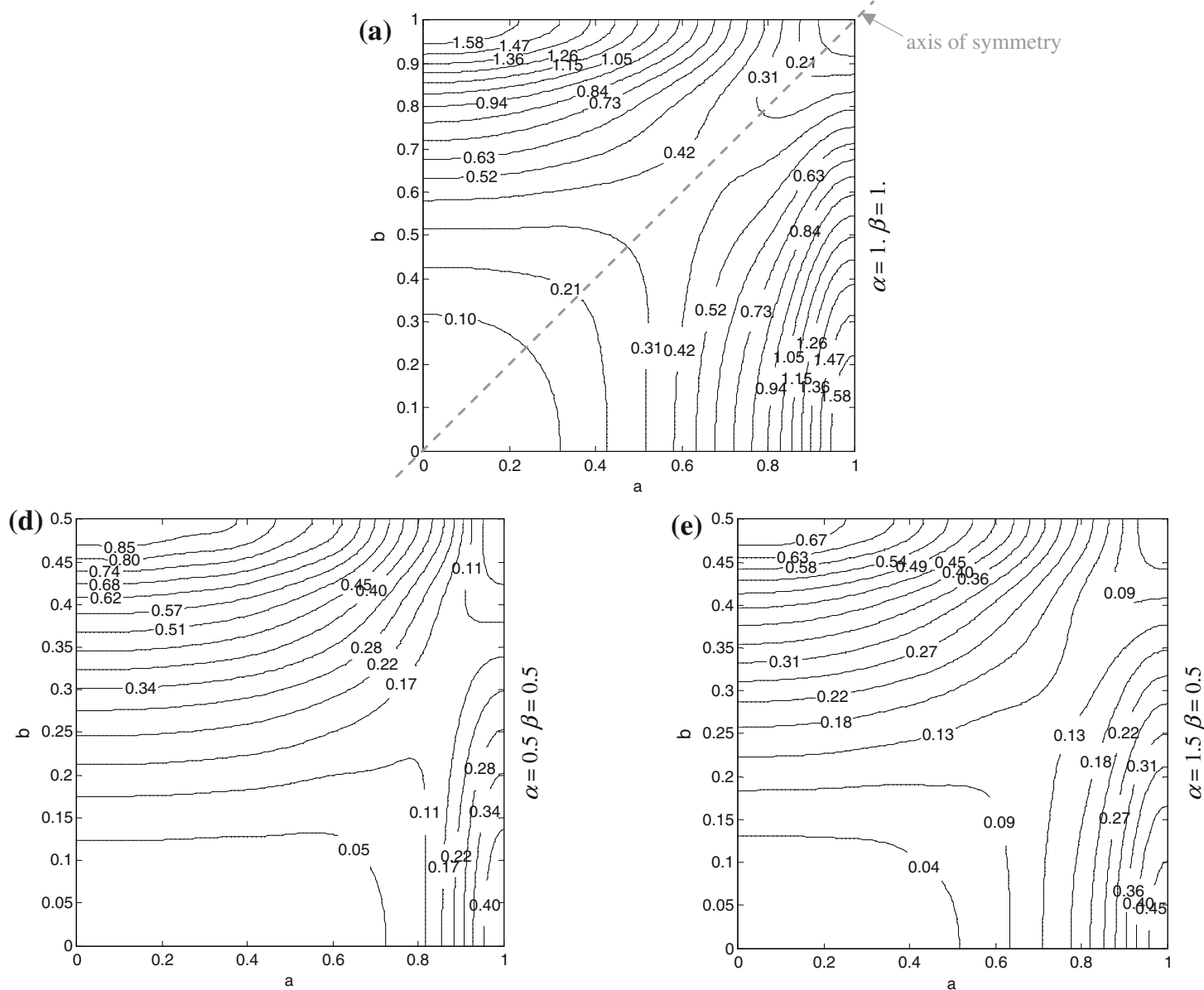

Fig. 4 Distribution patterns of strain energy densities $d U$ for three anisotropy degrees $(\alpha=0.5,1$ and 1.5$)$ and three aspect ratios $\left(\beta=0.5,1\right.$ and 1.5). Shear modulus $\mu_{x y}=100 \mathrm{MPa}$ and side $a=1 \times 10^{-2} \mathrm{~m}$

The proposed model involved a potential interest in the framework of material properties identification. The measurement of shear properties and especially out-of-plane components is challenging in composite structures or biological materials. In case of transversely isotropic material with isotropic plane coinciding with the cross-section (Fig. 1b: down-left corner), the inversion of Eq. (11) allowed obtaining shear modules $\mu_{x z}$ and $\mu_{x y}$ since $\alpha=1$ for a given torsion stiffness. For the same constitutive material but with the isotropic plane oriented perpendicular to the cross-section (Fig. 1b: up-right corner), it gave the out-of-plane shear modulus $\mu_{x z}$, replacing $\mu_{x y}$ by its previously determined value. The torsion stiffness should be obtained from 

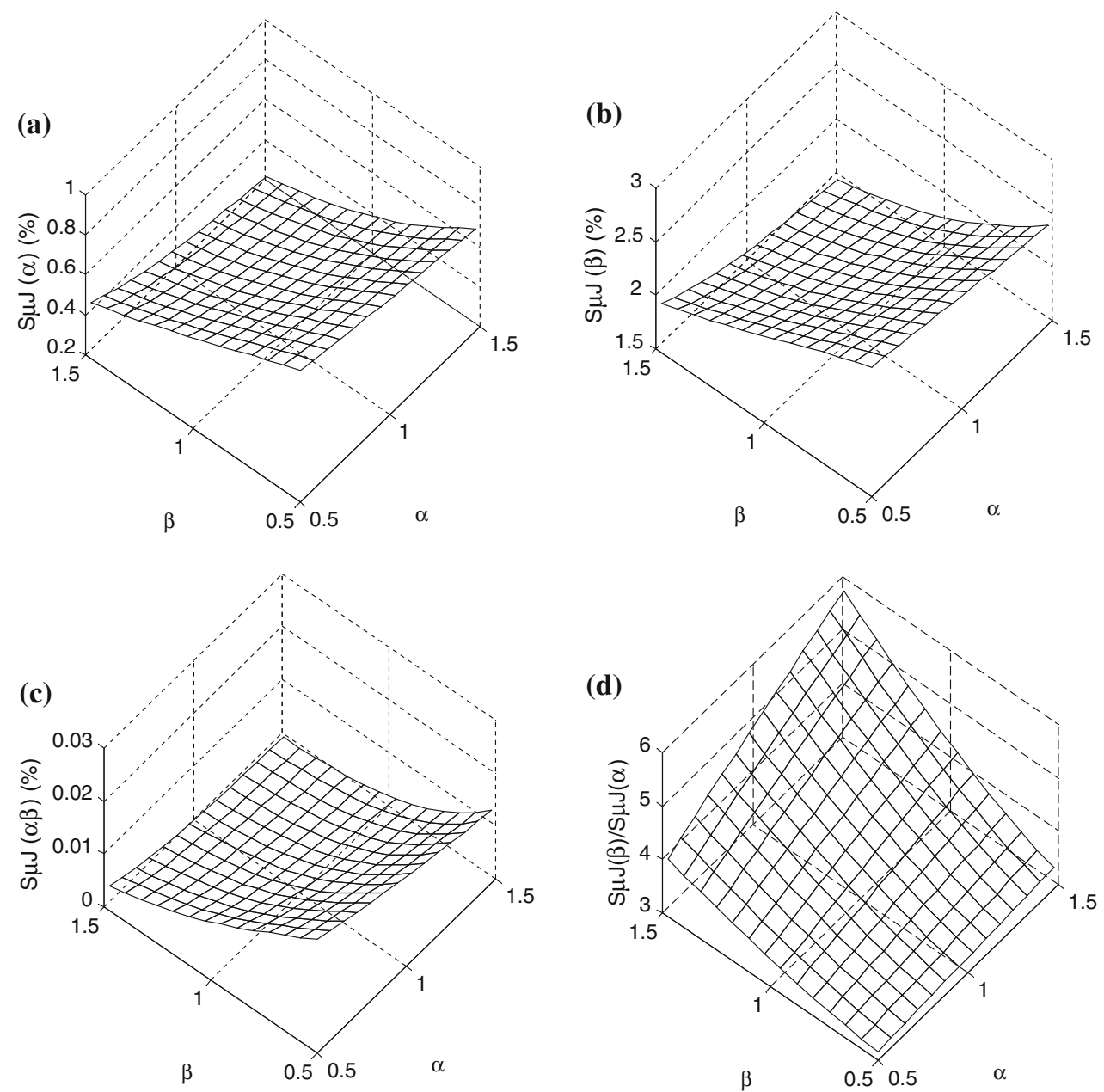

Fig. 5 Sensitivity of the relative variation of the torsion stiffness $\mu J$. a $S \mu J_{\alpha}$ : sensitivity to anisotropy degree $\alpha$; b $S \mu J_{\beta}$ : sensitivity to aspect ratio $\beta ; \mathbf{c} S \mu J_{\alpha \beta}$ : crossed sensitivity to $\alpha$ and $\beta ; \mathbf{d} S \mu J_{\beta} / S \mu J_{\alpha}$ : ratio of sensitivities. Results were established with $\delta \alpha=\delta \beta=1 \%$ in Eq. (12), shear modulus $\mu_{x y}=100 \mathrm{MPa}$ and side $a=1 \times 10^{-2} \mathrm{~m}$

(a) $\alpha=1 ; \beta=1$

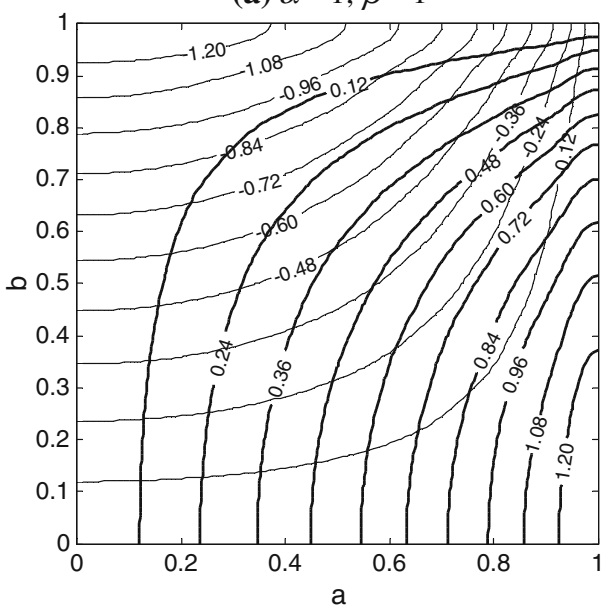

(b) $\alpha=0.5 ; \beta=0.5$

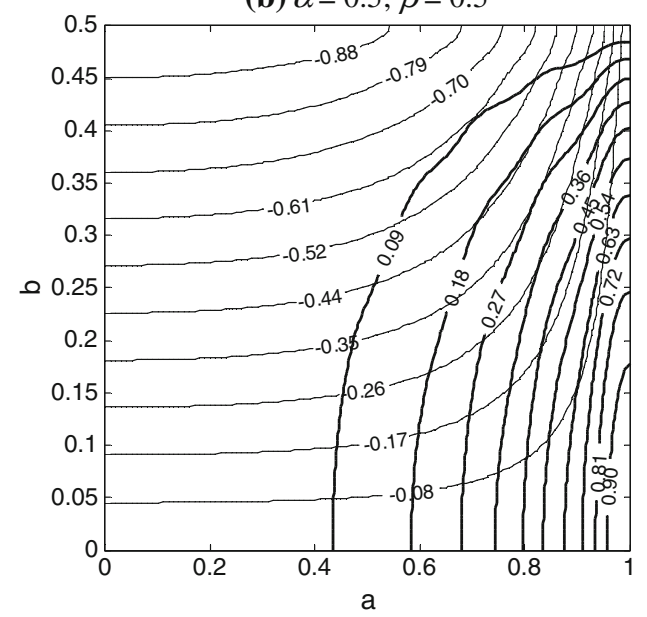

Fig. 6 Distribution patterns of stress fields $\left(\tau_{x y}, \tau_{x z}\right)$. a Square isotropic cross-section $(\alpha=1, \beta=1)$; b thin anisotropic cross-section $(\alpha=0.5, \beta=0.5)$. Shear modulus $\mu_{x y}=100 \mathrm{MPa}$, side $a=1 \times 10^{-2} \mathrm{~m}$ 
static torsion tests or modal analysis. As detailed Sect.2.2, sensitivities of torsion stiffness and shear modules showed similar tendencies, excepting their signs, since both were linearly dependant, the influence of crossed sensitivities being of order two. These results confirmed that the aspect ratio $\beta$ increased the reliability of the measurement process.

In conclusion, we proposed an analytical approach based upon an energy method to investigate the torsion problem of anisotropic cross-sections with simplified geometry. The evaluation was made with rectangular cross-sections involving two differentiated shear modules. The resolution was achieved using infinite trigonometric series to represent the stress function, and the process showed a fast convergence with a very good accuracy.

\section{Appendix}

$$
\begin{aligned}
S \mu J_{\alpha} & =\frac{\partial \mu J}{\partial \alpha} \frac{\delta_{\alpha}}{\mu J}=\frac{\partial f}{\partial \alpha} \frac{\delta_{\alpha}}{f}=\delta_{\alpha} \frac{\sum_{m} \sum_{n} 1 / m^{2}\left(\alpha \beta^{2} m^{2}+n^{2}\right)^{2}}{\sum_{m} \sum_{n} 1 / m^{2} n^{2}\left(\alpha \beta^{2} m^{2}+n^{2}\right)} \\
S \mu J_{\beta} & =\frac{\partial \mu J}{\partial \beta} \frac{\delta_{\beta}}{\mu J}=\frac{\partial f}{\partial \beta} \frac{\delta_{\beta}}{f}=\delta_{\beta} \frac{m \quad{ }_{n}\left(3 n^{2}+\alpha \beta^{2} m^{2}\right) / m^{2} n^{2}\left(\alpha \beta^{2} m^{2}+n^{2}\right)^{2}}{m{ }_{n} 1 / m^{2} n^{2}\left(\alpha \beta^{2} m^{2}+n^{2}\right)} \\
S \mu J_{\alpha \beta} & =\frac{\partial^{2} \mu J}{\partial \alpha \partial \beta} \times \frac{\delta_{\alpha} \delta_{\beta}}{\mu J}=\frac{\partial^{2} f}{\partial \alpha \partial \beta} \times \frac{\delta_{\alpha} \delta_{\beta}}{f}=\delta_{\alpha} \delta_{\beta} \frac{m{ }_{n}\left(3 n^{2}-\alpha \beta^{2} m^{2}\right) / m^{2}\left(\alpha \beta^{2} m^{2}+n^{2}\right)^{3}}{m \quad n m^{2} n^{2}\left(\alpha \beta^{2} m^{2}+n^{2}\right)}
\end{aligned}
$$

\section{References}

1. Allen, D.N.: Relaxation Methods. McGraw-Hill, London (1955)

2. Benveniste, Y.: A new approach to the application of Mori-Tanaka's theory in composite materials. Mech. Mat. 6, 147157 (1987)

3. Ely, J.F., Zienkiewicz, O.C.: Torsion of compounds bars—a relaxation solution. Int. J. Mech. Sci. 1, 356-365 (1960)

4. Estivalèzes, E., Couteau, B., Darmana, R.: 2D calculation method based on composite beam theory for the determination of local homogenized stiffnesses of long bones. J. Biomech. 34(2), 277-283 (2001)

5. Herrman, L.R.: Elastic analysis of irregular shaped prismatic beams by the method of finite elements. J. Eng. Mech. Div. Prc. ASCE 94(EM4), 965-983 (1968)

6. Horgan, C.O., Chan, A.M.: Torsion of functionally graded isotropic linearly elastic bars. J. Elast. 52, 181-199 (1999)

7. Lekhnitshii, S.G.: Theory of Elasticity of an Anisotropic Body. Mir Publishers, Moscow (1981)

8. Meirovitch, L.: Elements of Vibration Analysis. McGraw-Hill, New York (1975)

9. Manson, W.E., Herrman, L.R.: Elastic Analysis of Irregular Shaped Prismatic Beams by the Method of Finite Elements. Davis, Dept. Civ. Eng. University of California, Tech. Report no 67-1 (1967)

10. Muskhelishvilli, N.I.: Some Basic Problems of the Mathematical Theory of Elasticity. p. 561, Noordhoff, Groningen (1953)

11. Pereira, J.C.: Contribution à l'étude du comportement mécanique des structures en matériaux composites: caractéristiques homogénéisées des poutres composites, comportement dynamique des coques composites. PhD thesis, INSA Lyon, France (1995)

12. Rooney, F.J., Ferrari, M.: Tension, bending and flexure of functionally graded cylinders. Int. J. Solids Struct. 38, 413421 (2001)

13. Shaw, F.S.: The torsion of solid and hollow prisms in the elastic and plastic range by relaxation methods. Austral. Counc. Aeronaut., Sydney, 92 p, Report ACA-11, 38 (1944)

14. Sokolnikoff, I.S.: Mathematical Theory of Elasticity. McGraw-Hill, USA (1956)

15. Southwell, R.V.: Relaxation Methods in Theoretical Physics. p. 85 Clarendon press, Oxford (1946)

16. Swider, P., Jacquet-Richardet, G., Pereira, J.C.: Interactions between numerical and experimental approaches in composite structure dynamics. Compos. Struct. 43(2), 127-135 (1998)

17. Timoshenko, S., Goodier, J.N.: 1934 reissued 1987. Theory of Elasticity. McGraw-Hill, USA (1934)

18. Zienkiewicz, O.C., Cheung, Y.K.: Finite elements in the solution of field problems. Engineer 8, 507-510 (1965) 